\title{
Auditory localization and judgments of straight ahead during adaptation to prism
}

\author{
Samuel C. MeLaughlin and John L. Bower \\ TUFTS UNIVERSITY
}

\begin{abstract}
Abstraet
During adaptation to prismatic displacement, there are systematic changes in visual localization, in auditory localization, and in subjective straight ahead. The change in auditory localization is associated with the change in straight ahead rather than with the change in visual localization.

\section{Introduetion}

In a previous report from this laboratory (McLaughlin \& Rifkin, 1965), adaptation to prism was interpreted in terms of the concept of an egocentric frame of reference having "straight ahead"' as its primary direction of reference. The frame of reference provides a schema which $S$ uses in localizing objects with respect to himself. It was shown that the frame of reference and the visual display interact in two different ways to produce adaptive effect. In what will be referred to here as Type I adaptation, the entire frame of reference shifts, the visual target and the entire visual display being (so to speak) "carried along" in the direction of correct localization. In what will be referred to here as Type II adaptation, the visual target (along with the entire visual display) changes its position within the frame of reference-i.e., with respect to straight ahead.

In the present experiment, this theoretical model is applied to the study of auditory localization during adaptation to prism. The theory generates a clear-cut prediction: since straight ahead is "common to all sensory modalities" (McLaughlin \& Rifkin, 1965), any cross-modal transfer should occur by way of the change in straight ahead. If the experimental conditions can be arranged so that "straight ahead" and "visual" judgments follow different trends during the course of adaptation, "auditory" judgments should adhere to the trend of subjective straight ahead.

\section{Method}

The problem requires that the adaptive process be studied as an event over time, with rapid and precise measurements being taken repeatedly during the course of adaptation, and with the test procedure producing a minimum of interference with the process of adaptation. The method described by McLaughlin \& Rifkin (1965) was therefore employed.

\section{Apparatus}

The visual stimulus was a luminous rectangle $3 \mathrm{~cm}$ high and $0.2 \mathrm{~cm}$ wide with a uniform black surround. $S$ viewed it from a distance of $33 \mathrm{~cm}$, his head position being held constant with the aid of a bite board containing his dental impression. The auditory stimulus (intermittent white noise) was emitted from a concealed speaker directly above the visual stimulus. S's task was to move a brass pointer along a horizontal track perpendicular to his line of sight. The pointer was tapered upward, its tip being $0.2 \mathrm{~cm}$ lower than the bottom edge of the rectangle.

\section{Procedure}

At the start of the experiment, the display containing the visual and auditory stimuli was adjusted in lateral position so that the luminous rectangle appeared to $\mathrm{S}$ to be exactly straight ahead. The room was then darkened, the visual stimulus was turned off, and wedge prisms of 20 prism diopters were placed in front of S's eyes.

$\mathrm{S}$ was first instructed to set the pointer straight ahead. When that setting had been made and recorded, the auditory stimulus was turned on and $S$ was instructed to set the pointer directly beneath it. When that setting had been made and recorded, the sound was turned off, the luminous rectangle was turned on, and $S$ was instructed to set the pointer directly beneath the rectangle. When this third setting had been made and recorded, the display was illuminated, enabling $\mathrm{S}$ to see his hand and the pointer as well as the luminous rectangle. He was again instructed to set the pointer directly beneath the rectangle.

This sequence of four settings was repeated over and over, except that the visual stimulus (which had been left off during the first two settings) remained on during the rest of the experimental session. The session was continued until S's setting on the visual stimulus (in the dark) was in error by $0.5 \mathrm{~cm}$ or less.

The experiment was repeated with six Ss, each of whom was experienced in making observations of this type. ${ }^{2}$ These six were selected from an initial group of nine, the criterion of selection being accuracy in auditory localization. Each $S$ went through two experimental sessions: once with prismatic displacement to the right (as in Fig. 1), and once with prismatic displacement to the left. Only those data were used in which the initial "straight ahead" and "sound" settings were in error by less than $1.0 \mathrm{~cm}$. Each $\mathrm{S}$ repeated the experiment as many times as necessary until that criterion had been met.

\section{Results}

Figure 1 shows the settings made by one $\mathrm{S}$ in one experimental session. Gradual adaptation to displaced vision is represented by the gradual approach of "visual" settings toward zero. "Straight ahead" settings show an initial change that parallels the change in "visual" settings, then a shift back toward zero. Settings on the sound follow "straight ahead" settings closely. 


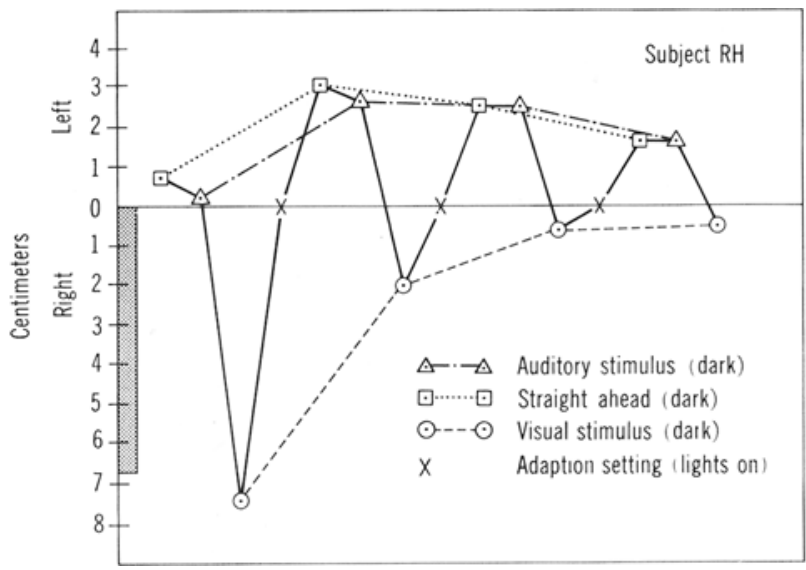

Fig. 1. Results for one S, one experimental session. Each point represents one setting of the pointer. The zero point on the ordinate represents the actual position of the visual and auditory stimuli. The thickened portion of the ordinate represents the direction and magnitude of the deviation induced by the prism. The solid line connects consecutive settings.

The data were analyzed to evaluate the tendency for "auditory" settings to follow "straight ahead" settings rather than "visual settings. This was done by comparing each "auditory" setting with (a) the "straight ahead" setting immediately preceding it, and (b) the "visual" setting immediately following it. The first three settings in each session were excluded from this analysis. For all 12 sessions, the mean difference between adjacent "straight ahead" and "auditory" settings was $0.16 \mathrm{~cm}$, which is not significantly different from zero $(S D=1.36 \mathrm{~cm}, t=1.16)$; and the mean difference between adjacent "auditory" and "visual" settings was $4.12 \mathrm{~cm}$, which is different from zero $(\mathrm{N}=72, \mathrm{SD}=0.546 \mathrm{~cm}, \mathrm{t}=7.55, \mathrm{p}<.0001)$.

\section{Discussion}

This finding - that auditory localization adheres to the trend of straight ahead settings-indicates that a change in auditory localization takes place during Type I adaptation but not during Type II adaptation. It also suggests that the shift in auditory localization may not be present except at an intermediate stage in the adaptive process, for in complete adaptation the perception of straight ahead (and consequently the perception of auditory direction) would be veridical. Thus, in an experiment such as that reported by Held \& Bossom (1961), in which Ss had extensive adaptive exposure involving active locomotion while wearing prisms, measurements taken before and after adaptation might show no shift in auditory localization.

The presence of a shift in auditory localization and a matching shift in "straight ahead" at an intermediate stage of adaptation is corroborated in an experiment reported by Harris (1963). Measurements of visual, auditory, and "straight ahead" localization were made before and after an adaptive exposure consisting of 90 pointing gestures with hand seen through prism. The result was a shift in auditory localization amounting to $44 \%$ of the prism deviation and a shift in straight ahead amounting to $48 \%$ of the prism deviation. That Harris' adaptive procedure did in fact produce an intermediate degree of adaptation rather than complete adaptation to prism is confirmed beyond any doubt by the observation that the primary adaptive change-the shift in visual localization-was only $50 \%$ of the deviation induced by the prism. In terms of the theoretical model employed here, the Harris experiment is particularly valuable in that it demonstrates a technique for eliciting Type I adaption in relatively pure form.

In the 1963 report, Harris correctly stated his conclusions in terms of "this rapid adaptation to displaced vision," implying an awareness that they might not be applicable to the fully adapted state. More recently, however, he has stated that "the shift in pointing 'straight ahead' is just as large as the shift in pointing at visual targets" (Harris, 1965); and this statement is clearly intended to apply, not to the semi-adapted condition, but to experiments in which "a biteboard keeps $\mathrm{S}$ from moving his head while adapting." The results presented here, as well as those reported in an earlier paper (McLaughlin \& Rifkin, 1965) indicate that the ratio between visual shift and "straight ahead" shift varies over a wide range during the course of adaptation. Harris' finding that the two are equal is evidently attributable to his use of an adaptation procedure which produces a semi-adapted condition, combined with his use of an experimental method (taking measurements before and after an arbitrary amount of adaptive exposure) which treats prism adaptation as a static condition rather than as a dynamic process.

These results suggest that the theoretical model advanced here may have predictive value for other aspects of the problem of specificity and transfer of adaptive effect. That possibility is being investigated.

\section{References}

HARRIS, C. S. Adaptation to displaced vision: visual, motor, or proprioceptive change? Science, 1963, 140, 812-813.

HARRIS, C. S. Comment on "Change in straight ahead during adaptation to prism." Psychon. Sci., 1965, 2, 285-286.

HELD, R., \& BOSSOM. J. Neonatal deprivation and adult rearrangement: complimentary techniques for analyzing plastic sensorymotor coordination. J. comp. physiol. Psychol., 1961, 54, 33-37. MCLAUGHLIN, S. C., \& RIFKIN, K. I. Change in straight ahead during adaptation to prism. Psychon. Sci., 1965, 2, 107-108.

Notes

1. This research supported in part by U.S. Public Health Service Research Grant NB-04540-03 from NIH, Div. Neurol. Diseases and Blindness; and in part by a contract from the Behavioral Sciences Research Branch of the U.S. Army Medical Research \& Development Command.

2. Two of the six Ss, when relatively unpracticed, gave results in which the "sound" settings did not follow "straight ahead" settinss as closely as in Fig. 1, though both kinds of settings showed an initial shift from zero followed by a return toward zero. These preliminary results (not included in the summary data reported here) may be significant for some interpretations of the present finding. 